\title{
Does otitis media in early childhood affect later behavioural development? Results from the Western Australian Pregnancy Cohort (Raine) Study
}

\section{Authors:}

Cheryl Da Costa ${ }^{1,2,3}$

Robert H. Eikelboom ${ }^{2,3,4}$

Angela Jacques 5

De Wet Swanepoel ${ }^{2,3,4}$

Andrew J.O. Whitehouse ${ }^{6}$

Sarra E. Jamieson ${ }^{6}$

Christopher G. Brennan-Jones ${ }^{2,3,6,7}$

1. Faculty of Medicine, Dentistry and Health Sciences, The University of Western Australia, Perth, Australia

2. Ear Science Institute Australia, Perth, Australia

3. Ear Sciences Centre, The University of Western Australia, Perth, Australia

4. Department of Speech-Language Pathology and Audiology, University of Pretoria, Pretoria, South Africa

5. School of Population and Global Health, The University of Western Australia, Perth, Australia

6. Telethon Kids Institute, The University of Western Australia, Perth, Australia

7. Department of Audiology, Perth Children's Hospital, Perth, Australia

Key words: otitis media, behaviour, child development, Raine Study

Abbreviations: Otitis Media (OM), rOM (Recurrent Otitis Media), Child Behaviour Checklist (CBCL)

\section{Contact details:}

Cheryl Da Costa

The University of Western Australia

35 Stirling Hwy, Crawley WA 6009

0411368343

cheryl.c.dacosta@gmail.com 


\section{Abstract}

Objectives: To examine the relationship between early life episodes of otitis media and later behavioural development with adjustment for confounders.

Design: Longitudinal cohort study.

Setting: The Western Australian Pregnancy Cohort (Raine) Study recruited 2900 pregnant women from King Edward Memorial Hospital (KEMH) in Perth, Western Australia, between 1989 and 1991.

Participants: Data from the children born were collected at both the Year 3 and Year 5 follow up. At Year 3, $n=611$ were diagnosed with recurrent otitis media through parent-report and clinical examination. At Year 5, $n=299$ were considered exposed to otitis media based upon tympanometry results.

Main Outcome Measures: Performance in the Child Behaviour Checklist (CBCL), a questionnaire completed by the primary caregiver at Year 10.

Results: Significant associations were found between recurrent otitis media at Year 3 and internalising behaviours $(P=0.011)$, and the somatic $(P=0.011)$, withdrawn $(P=$ $0.014)$, attention $(P=0.003)$ and thought problems domains $(P=0.021)$, and the total CBCL score $(P=0.010)$. A significant association was also found between exposure to otitis media at Year 5 and externalising behaviours $(P=0.026)$.

Conclusions: A modest association was seen between recurrent otitis media at Year 3 and exposure to otitis media at Year 5 and a number of behaviour domains at Year 10. 


\section{Introduction}

Otitis media $(\mathrm{OM})$ is a general term used to describe conditions that involve the inflammation of the middle ear cleft and is highly prevalent in early childhood, affecting up to $90 \%$ of children prior to school entry. ${ }^{1,2}$ Effusion associated with OM and the resultant hearing deficit has been shown to compromise language development, which can, in turn, influence the behaviour of affected children. ${ }^{3}$ However, behavioural development is a multifactorial process. Therefore it has been difficult to establish a direct association between $\mathrm{OM}$ and poor behavioural development, without the influence of confounding factors. The aim of this longitudinal investigation was to examine the relationship between early life episodes of $\mathrm{OM}$ and poor behavioural development with adjustment for potential prenatal, postnatal and environmental confounders. The underlying hypothesis was that the presence of OM would influence the development of behavioural problems in later childhood, including a higher prevalence of conduct problems, hyperactivity and attention difficulties. ${ }^{3-5}$

It should be noted that previous studies in this area have been restricted in their application to clinical practice due to a number of design limitations, including small sample size, ${ }^{6,7}$ purely subjective detection of $O M,{ }^{3,8}$ selection bias, ${ }^{4,9}$ and the presence of multiple confounding factors. ${ }^{8,10-13}$ This study sought to address these limitations, with the intention of producing findings to better inform our approach to management and intervention for children with OM. 


\section{Methods}

\section{Ethical Considerations}

Human ethics committees at both King Edward Memorial Hospital (KEMH) and Princess Margaret Hospital in Perth, Western Australia, approved participant recruitment and follow-up for the Western Australian Pregnancy Cohort (Raine) Study. Informed written consent was obtained from every pregnant woman and consent was also obtained at each follow up stage from the guardian of each child participant. Each child participant was then re-consented at age 18 years for the use of stored data. All data provided was de-identified prior to receipt.

\section{Raine Study Participants}

This study is based on data from the Raine Study, a longitudinal cohort study of 2900 pregnant women who volunteered to be a part of a study at $\mathrm{KEMH}$, the sole tertiary maternity hospital in Western Australia. These women were recruited from various private and public hospitals within Perth, Western Australia, between the months of May 1989 and November $1991 .{ }^{14}$ To be eligible for participation, these women were required to have a gestational age between 16 and 20 weeks, proficiency in English language sufficient enough to understand the implications of participation, the expectation to deliver their baby at $\mathrm{KEMH}$, and the intention to continue residency within Western Australia. ${ }^{14}$ The 2868 live born children from these pregnancies have been assessed over the past 28 years with respect to a number of factors. These included middle ear pathology, ${ }^{2,15}$ and behavioural characteristics. ${ }^{2,16}$

\section{Variables}

\section{Diagnostic Criteria}

The presence of OM in the Raine Study was identified through a number of different methods. Two sample populations were selected based upon different identification 
criteria, each with their own strengths and limitations. The first sample included those children within the Raine Study cohort that had a recurrent history of OM (rOM) at the Year 3 follow-up, when children were approximately three years of age. ${ }^{2}$ These children were identified by a positive answer to the question, 'has your child ever in his/her life [had] otitis media (middle ear infection)? If so, how many?' If a participant was reported as having three or more episodes of $\mathrm{OM}$ in the first three years of life, they were considered to have a diagnosis of $\mathrm{rOM}^{2}$ If clinical examination by a specialist nurse revealed scarred, retracted, inflamed or perforated tympanic membranes, the presence of middle ear effusion or ventilation tubes in situ during the Year 1 - Year 3 follow-ups, they were also said to have a diagnosis of OM and included in this sample population, ${ }^{2}(n=611)$.

The second sample included those children that underwent a otoscopic examination with tympanometry at the Year 5 follow-up assessment, when children were approximately six years of age $\mathrm{e}^{15,17}$ the earliest time point in which this type of assessment was completed in this cohort. Type B tympanograms provide a measure of the presence of middle ear effusion, which was used to indicate exposure to OM (either acute $\mathrm{OM}$ or $\mathrm{OM}$ with effusion). ${ }^{15}$ Either unilateral or bilateral Type B tympanograms were included in this cohort $(n=299)$. Note that a definitive diagnosis of OM cannot be made using cross sectional data.

\section{Outcome Measure}

The outcome measure for this study was the Child Behaviour Checklist (CBCL) score, an empirically validated measure of child behaviour for use in children aged 418 years. This was completed by the primary caregiver at the Year 10 follow-up, when participants were approximately 10 years of age. ${ }^{16}$ The CBCL is a commonly used dimensional rating scale for emotional and behaviour problems in children that 
has been shown to be a good predictor of functional and behavioural impairment later in life, ${ }^{16}$ with good internal reliability and validity across a range of populations. ${ }^{16}$ The CBCL is a questionnaire consisting of 118 questions that are based on specific problem behaviours that may have been observed in the past 6 months. It is scored using a 3-point Likert Scale where 0 indicates the behaviour is absent; 1 indicates that the behaviour occurs sometimes; and 2 indicates that it occurs often. ${ }^{18}$ Performance in the CBCL is calculated using a total $t$-score (standardised by age and gender), with results apportioned across a number of relevant behavioural domains including withdrawn, somatic complaints (such as abdominal pain), anxious/depressed, social problems, thought problems, attention problems, delinquent behaviour, and aggressive behaviour. Some of these domains are also summarised under two behavioural subscales, internalising behaviours (problems within oneself) that include the somatic, withdrawal and anxious depressed domains, and externalising behaviours (conflicts with others or against societal expectations) that include the delinquency and aggression domains. Note that the $t$-scores, as opposed to raw scores, were used in this analysis.

\section{Potential Confounders}

In order to minimise confounding bias, a number of potential risk factors for OM, identified through the literature, were accounted for in the analyses. ${ }^{2}$ These risk factors are listed in Tables 1 and 2.

\section{Statistical Methods}

The prevalence of the predicted risk factors within both the rOM in the Year 3, and exposure to $\mathrm{OM}$ in the Year 5, sample populations was determined using frequency distributions (See Tables 1 and 2). Multiple linear regression models were then used to compare the continuous CBCL data across a number of domains at Year 10 as the 
dependent variable, against all the possible risk factors, alongside rOM at Year 3 and exposure to $\mathrm{OM}$ at Year 5 as the independent variables (See Tables 3 and 4). For all analyses, $\mathrm{p}<0.05$ was considered statistically significant. Data was analysed using IBM SPSS Statistics for Macintosh, Version 23.0 (Armonk, NY: IBM Corp).

\section{Results}

The overall prevalence of rOM at Year 3 was 26.8\% (611/2277), and 22.3\% (299/1343) for exposure to OM at Year 5. Of those with a rOM at Year 3, 85.4\% (522/611) had CBCL data recorded at Year 10, and of those exposed to OM at Year 5, 88.6\% (265/299) had CBCL data recorded at Year 10. Of those without rOM at Year 3, 81.6\% (1349/1666) had CBCL data recorded at Year 10, and of those that were not exposed to OM at Year 5, 89.6\% (935/1044) had CBCL data recorded at Year 10. A comprehensive range of potential risk factors for OM were available in the Raine Study and the prevalence of these within both Year 3 and Year 5 sample populations are displayed in Tables 1 and 2 respectively.

When using multiple linear regression analyses to estimate the predicted change in CBCL scores associated with the presence of $\mathrm{rOM}$ at Year 3, it was found that there was a statistically significant association between $\mathrm{rOM}$ at $\mathrm{Year} 3$ and internalising behaviours, the somatic complaints domain, the withdrawn domain, the attention problems domain, and the thought problems domain of CBCL scores, as well as the total CBCL score, after controlling for the effects of all other predictors in the model (Table 3).

When using multiple linear regression analyses to estimate the predicted change in CBCL scores associated with exposure to $\mathrm{OM}$ at Year 5, it was found that there was a statistically significant association between the exposure to $\mathrm{OM}$ at Year 5 and 
externalising behaviours after controlling for the effects of all other predictors in the model (Table 4).

\section{Discussion}

The analysis demonstrates a modest association between early life episodes of OM and behavioural development in later childhood as determined through CBCL data, a standardised measure of behaviour. The association differs between each group with $\mathrm{rOM}$ at Year 3 being associated with a greater range of behavioural problems measured at Year 10 in comparison with exposure to $\mathrm{OM}$ at Year 5. This may be a reflection of the nature of $\mathrm{OM}$ identification in this population, as those children with recurrent episodes of OM over a three-year period were selected, as opposed to the Year 5 recruitment cohort, which was cross-sectional. Recurrent OM is more strongly associated with an extended loss of hearing sensitivity, which is more likely to lead to problematic behaviour such as hyperactivity and attention deficit. ${ }^{2,3,19}$ Parental reporting is also more likely to capture the presence of more serious disease, as parents are more likely to recall events of greater chronicity and severity. ${ }^{3}$ Furthermore, as the data were collected at a younger age, episodes of OM in this sample population are more likely to be captured during the critical period of language development, which is said to be during the first few years of life. ${ }^{13,19,20}$ If hearing is disrupted during this critical period, the cognitive areas associated with auditory processing, speech and language may be affected, resulting in a greater chance of developing the behavioural problems observed due to the inability to communicate effectively.

Though exposure to $\mathrm{OM}$ at Year 5 was determined through a more objective means in comparison with parental report, ${ }^{21}$ it does not provide clear information with respect to either the severity or time course of the condition. This is because the data itself is 
based upon a single point in time. Though the presence of effusion can be indicative of chronicity, with the average duration of OM with middle ear effusion said to be three months and likely to recur, ${ }^{21}$ this cannot be said for certain within this population without trajectory analysis. This method of diagnosis is also at risk of under diagnosing the prevalence of $\mathrm{OM}$ in this population, which may underestimate the extent of any effects seen. Nevertheless, the results still demonstrate a significant association with externalising behaviours overall indicating that exposure of OM, irrespective of whether the exposure is prolonged, is still a sensitive variable. This is in line with studies that state that the severity and duration of episodes of OM are inconsequential to the development of behavioural sequelae, ${ }^{22}$ though it may contribute to the severity of the deficit seen.

It was interesting to note that $\mathrm{rOM}$ at Year 3 was associated with internalising behaviours, whereas exposure to $\mathrm{OM}$ at Year 5 was associated with externalising behaviours. It is suggested that the development of externalising behaviours is more strongly associated with a hearing deficit, whereas internalising behaviours is more strongly associated with chronic disease of any causation. ${ }^{3}$ This can be related back to the results obtained as the presence of $\mathrm{OM}$ is associated with an average hearing loss of between 20 and 30 decibels $(\mathrm{dB}){ }^{21}$ As such, identification through tympanometry may have captured more participants with a confirmed hearing deficit compared with parental report. This may have contributed to the association with externalising behaviours observed. In addition, rOM is related to recurrent episodes of $\mathrm{OM}$, a variable more strongly associated with chronic disease. As a result, these children with recurrent $\mathrm{OM}$ may have been more likely to develop internalising behaviours later in life. Nevertheless, it is thought that such dichotomous terms for behaviour are actually misleading as a single individual can display both types of behaviour concurrently. ${ }^{23,24}$ Therefore, the association with behavioural deficits as a whole was 
considered for the purposes of this study, rather than a focus on specific behavioural domains.

\section{Strengths and Limitations}

This investigation was embedded within the Raine Study, which enabled the inclusion of a large and prospectively studied participant cohort. The wealth of data collected has ensured that a wide range of potential risk factors associated with the development of OM could be identified and accounted for within the analysis. This helped determine the extent to which OM was associated with behaviour without the influence of confounders. ${ }^{8,10-13}$ Similarly, the use of the Raine Study data has allowed for a larger, population-based sample size to be analysed, increasing the external validity of the data. Another strength of this study is the use of CBCL to identify behavioural deficits. The CBCL has demonstrated strong internal consistency in the identification of psychopathology in children, and is also deemed comparable with other studies that describe behavioural deficits in childhood. ${ }^{16}$ Although there is the potential for observer bias as CBCL data is based upon parental perspective of behaviour, which could be affected by the circumstances of the individual parent, ${ }^{23}$ the use of this data as the primary outcome measure has been validated, ${ }^{16}$ particularly for the prediction of child and adolescent mental health outcomes. ${ }^{18}$

A number of limitations in this study should also be considered when interpreting the results. Though many risk factors were identified and accounted for in the multiple linear regression models, not all risk factors could be included due to a lack of data. For example, a family history of OM, pacifier use, upper respiratory tract infection, and snoring have all been linked with the development of OM across a wide range of studies, ${ }^{2}$ yet were not available through the Raine Study. The issues associated with the identification of $\mathrm{OM}$ were also an important limitation, in particular the potential 
for recall bias through the use of parental report. Despite this possibility, it should be noted that the sensitivity and specificity of rOM has been calculated as between 75 $95 \%$ and $65-100 \%$ respectively, ${ }^{25}$ with parents more likely to underestimate the number of episodes a child has experienced. ${ }^{2}$ The ideal method would be for all those children identified to be subject to clinical confirmation with either tympanometry or pneumatic otoscopy, and continuous monitoring over a set time period to determine the extent and chronicity of the disease. ${ }^{2}$ However, the resources required to achieve this were not available in this study.

\section{Conclusions}

This study has shown that a modest association exists between rOM at Year 3 and exposure to $\mathrm{OM}$ at Year 5 and several behavioural domains in later childhood, when controlling for confounders. Though this association is minor across all domains, it is interesting to note that an association with behaviour still exists with respect to OM, between 5 to 7 years after the condition was first recorded. These associations are often diminished over time due to the natural accumulation of other sources of variance. $^{3}$ As the presence of behavioural issues in childhood has a strong influence upon later mental health outcomes, this finding could create a target population for the monitoring of key language milestones and subsequent early intervention if deficits become apparent. ${ }^{16,19,24}$ 


\section{ACKNOWLEDGEMENTS}

The Raine Study is funded by the Raine Medical Research Foundation, the National Health and Medical Research Council (NHMRC), The University of Western Australia, Curtin University, Edith Cowan University, The Telethon Kids Institute, and the Women and Infants Research Foundation. We are extremely grateful to all the participants and their families who took part in the Raine Study, Jenny Mountain (Raine Study Manager) and the whole Raine Study team, which includes data collectors, cohort managers, data managers, clerical staff, research scientists and volunteers. AJOW is funded by a Senior Research Fellowship from the NHMRC (\#1077966); CGBJ is funded by a Health Professional Research Fellowship from the NHMRC (\#1142897).

\section{CONFLICT OF INTEREST}

None to declare. 
Table 1: Characteristics of the study population by presence of rOM at Year 3

\begin{tabular}{|c|c|c|c|c|}
\hline \multirow[t]{2}{*}{ Risk Factors } & \multicolumn{2}{|c|}{$\underline{\mathrm{rOM}}$ at Year $3(n=611)$} & \multicolumn{2}{|c|}{ No rOM $(n=1666)$} \\
\hline & $n$ & $\%$ & $n$ & $\%$ \\
\hline \multicolumn{5}{|l|}{ Male Gender } \\
\hline Yes & 327 & 53.5 & 827 & 49.6 \\
\hline No & 284 & 46.5 & 839 & 50.4 \\
\hline \multicolumn{5}{|c|}{ Mother Spoke Language Other than English } \\
\hline Yes & 23 & 3.8 & 100 & 6.0 \\
\hline No & 588 & 96.2 & 1566 & 94.0 \\
\hline \multicolumn{5}{|l|}{ Maternal Ethnicity } \\
\hline Caucasian & 585 & 95.7 & 1462 & 87.8 \\
\hline Other & 26 & 4.3 & 204 & 12.2 \\
\hline \multicolumn{5}{|c|}{ Household Income Below Poverty Line } \\
\hline$<$ AU $\$ 24,000$ per annum & 225 & 36.8 & 636 & 38.2 \\
\hline$>$ AU\$24,000 per annum & 361 & 63.2 & 952 & 61.8 \\
\hline \multicolumn{5}{|l|}{ Low Maternal Education } \\
\hline$<$ Year 12 & 343 & 56.1 & 945 & 56.7 \\
\hline$>$ Year 12 & 254 & 43.9 & 686 & 43.3 \\
\hline \multicolumn{5}{|l|}{ Passive Smoking } \\
\hline Yes & 215 & 35.2 & 562 & 33.7 \\
\hline No & 316 & 64.8 & 839 & 66.3 \\
\hline \multicolumn{5}{|l|}{ Parity } \\
\hline No older siblings & 256 & 41.9 & 836 & 50.2 \\
\hline One or more older siblings & 355 & 58.1 & 829 & 49.8 \\
\hline \multicolumn{5}{|c|}{ Prematurity ( $<37$ week gestation) } \\
\hline Yes & 47 & 7.7 & 143 & 8.6 \\
\hline No & 552 & 92.3 & 1483 & 91.4 \\
\hline \multicolumn{5}{|l|}{ Breastfeeding Stopped } \\
\hline$<6$ months & 294 & 48.1 & 702 & 42.1 \\
\hline$>6$ months & 285 & 51.9 & 837 & 57.9 \\
\hline \multicolumn{5}{|l|}{ Low birth weight $(<2500$ g) } \\
\hline Yes & 48 & 7.9 & 144 & 8.6 \\
\hline No & 561 & 92.1 & 1517 & 91.4 \\
\hline \multicolumn{5}{|c|}{ Alcohol in Pregnancy (at 34 week gestation) } \\
\hline Once a week or more & 252 & 41.2 & 574 & 34.5 \\
\hline Zero Alcohol & 319 & 58.8 & 960 & 65.5 \\
\hline \multicolumn{5}{|l|}{ Daycare Attendance } \\
\hline Yes & 346 & 56.6 & 739 & 44.4 \\
\hline No & 99 & 43.4 & 375 & 55.6 \\
\hline \multicolumn{5}{|l|}{ Introduction of Other Milk } \\
\hline$<6$ months & 435 & 71.2 & 1087 & 65.2 \\
\hline$>6$ months & 142 & 28.8 & 441 & 34.8 \\
\hline \multicolumn{5}{|l|}{ Asthma } \\
\hline Yes & 95 & 15.5 & 195 & 11.7 \\
\hline No & 516 & 84.5 & 1471 & 88.3 \\
\hline \multicolumn{5}{|l|}{ Allergies } \\
\hline Yes & 188 & 30.8 & 348 & 20.9 \\
\hline No & 322 & 69.2 & 946 & 79.1 \\
\hline
\end{tabular}


Table 2: Characteristics of the study population by exposure to OM at Year 5

\begin{tabular}{|c|c|c|c|c|}
\hline \multirow[t]{2}{*}{ Risk Factors } & \multicolumn{2}{|c|}{ OM at Year $5(n=299)$} & \multicolumn{2}{|c|}{ No OM $(n=1044)$} \\
\hline & $n$ & $\%$ & $n$ & $\%$ \\
\hline \multicolumn{5}{|l|}{ Male Gender } \\
\hline Yes & 136 & 45.5 & 471 & 45.1 \\
\hline No & 163 & 54.5 & 570 & 54.6 \\
\hline \multicolumn{5}{|c|}{ Mother Spoke Language Other than English } \\
\hline Yes & 273 & 91.3 & 994 & 95.5 \\
\hline No & 26 & 8.7 & 47 & 4.5 \\
\hline \multicolumn{5}{|l|}{ Maternal Ethnicity } \\
\hline Caucasian & 257 & 86.0 & 957 & 91.9 \\
\hline Other & 42 & 14.0 & 84 & 8.1 \\
\hline \multicolumn{5}{|c|}{ Household Income Below Poverty Line } \\
\hline$<$ AU $\$ 24,000$ per annum & 114 & 38.1 & 370 & 35.5 \\
\hline$>$ AU $\$ 24,000$ per annum & 171 & 57.2 & 625 & 64.5 \\
\hline \multicolumn{5}{|l|}{ Low Maternal Education } \\
\hline$<$ Year 12 & 159 & 53.2 & 574 & 55.1 \\
\hline$>$ Year 12 & 133 & 44.5 & 451 & 44.9 \\
\hline \multicolumn{5}{|l|}{ Passive Smoking } \\
\hline Yes & 103 & 34.4 & 315 & 30.3 \\
\hline No & 148 & 49.5 & 573 & 69.7 \\
\hline \multicolumn{5}{|l|}{ Parity } \\
\hline No older siblings & 142 & 47.5 & 455 & 43.7 \\
\hline One or more older siblings & 156 & 52.2 & 586 & 56.3 \\
\hline \multicolumn{5}{|c|}{ Prematurity ( $<37$ weeks gestation) } \\
\hline Yes & 19 & 6.4 & 72 & 6.9 \\
\hline No & 273 & 91.3 & 948 & 93.1 \\
\hline \multicolumn{5}{|c|}{ Breastfeeding Stopped $<6$ months } \\
\hline Yes & 156 & 52.2 & 439 & 42.2 \\
\hline No & 125 & 41.8 & 559 & 57.8 \\
\hline \multicolumn{5}{|l|}{ Low birth weight $(<2500 \mathrm{~g})$} \\
\hline Yes & 21 & 7.0 & 75 & 7.2 \\
\hline No & 278 & 93.0 & 963 & 92.8 \\
\hline \multicolumn{5}{|c|}{ Alcohol in Pregnancy (at 34 week gestation) } \\
\hline Once a week or more & 105 & 35.1 & 399 & 38.3 \\
\hline Zero Alcohol & 173 & 57.9 & 569 & 61.7 \\
\hline \multicolumn{5}{|l|}{ Daycare Attendance } \\
\hline Yes & 132 & 44.1 & 510 & 49.0 \\
\hline No & 77 & 25.8 & 213 & 51.0 \\
\hline \multicolumn{5}{|l|}{ Introduction of Other Milk } \\
\hline$<6$ months & 190 & 63.5 & 798 & 76.7 \\
\hline$>6$ months & 91 & 30.4 & 283 & 23.3 \\
\hline \multicolumn{5}{|l|}{ Asthma } \\
\hline Yes & 37 & 12.4 & 137 & 13.2 \\
\hline No & 262 & 87.6 & 904 & 86.8 \\
\hline \multicolumn{5}{|l|}{ Allergies } \\
\hline Yes & 73 & 24.4 & 252 & 24.2 \\
\hline No & 168 & 56.2 & 599 & 75.8 \\
\hline
\end{tabular}


Table 3: Output of standard multiple regression analyses comparing the effect of rOM at Year 3 upon CBCL scores adjusting for all potential risk factors

\begin{tabular}{lccccc}
\hline & $\beta$ & $\mathrm{SE}(\beta)$ & $P$ & \multicolumn{2}{c}{$95 \% \mathrm{CI}(\beta)$} \\
\hline Total Score & 2.03 & 0.79 & $\mathbf{0 . 0 1 0} *$ & 0.48 & 3.57 \\
Internalising & 1.84 & 0.72 & $\mathbf{0 . 0 1 1} *$ & 0.42 & 3.26 \\
Externalising & 1.37 & 0.75 & 0.069 & -0.11 & 2.84 \\
Delinquent & 0.32 & 0.45 & 0.472 & -0.59 & 1.20 \\
Anxious/Depressed & 0.80 & 0.42 & 0.058 & -0.03 & 1.62 \\
Somatic & 1.28 & 0.50 & $\mathbf{0 . 0 1 1} *$ & 0.30 & 2.25 \\
Withdrawn & 0.96 & 0.39 & $\mathbf{0 . 0 1 4} *$ & 0.20 & 1.72 \\
Attention & 1.38 & 0.46 & $\mathbf{0 . 0 0 3 *}$ & 0.48 & 2.28 \\
Aggression & 0.64 & 0.43 & 0.140 & -0.21 & 1.49 \\
Thought Problems & 0.90 & 0.39 & $\mathbf{0 . 0 2 1} *$ & 0.14 & 1.66 \\
Social Problems & 0.82 & 0.45 & 0.072 & -0.07 & 1.70 \\
\hline
\end{tabular}


Table 4: Output of standard multiple regression analyses comparing the effect of exposure to OM at Year 5 upon CBCL scores adjusting for all potential risk factors

\begin{tabular}{lccccc}
\hline & $\beta$ & $\mathrm{SE}(\beta)$ & $P$ & \multicolumn{2}{c}{$95 \% \mathrm{CI}(\beta)$} \\
\hline Total Score & 1.50 & 1.07 & 0.163 & -0.61 & 3.60 \\
Internalising & 0.85 & 1.00 & 0.395 & -1.12 & 2.82 \\
Externalising & 2.31 & 1.03 & $\mathbf{0 . 0 2 6}$ & 0.28 & 4.33 \\
Delinquent & 0.83 & 0.62 & 0.178 & -0.38 & 2.04 \\
Anxious/Depressed & 0.53 & 0.57 & 0.353 & -0.59 & 1.64 \\
Somatic & -0.80 & 0.67 & 0.231 & -2.12 & 0.51 \\
Withdrawn & 0.79 & 0.55 & 0.148 & -0.28 & 1.86 \\
Attention & -0.04 & 0.61 & 0.948 & -1.23 & 1.15 \\
Aggression & 0.72 & 0.58 & 0.216 & -0.42 & 1.86 \\
Thought Problems & -0.08 & 0.52 & 0.876 & -1.10 & 0.93 \\
Social Problems & 0.29 & 0.61 & 0.631 & -0.90 & 1.48 \\
\hline
\end{tabular}




\section{References}

1. Kong K, Coates HL. Natural history, definitions, risk factors and burden of otitis media. Med J Aust. 2009 Nov 2;191(9 Suppl):S39-43

2. Brennan-Jones CG, Whitehouse AJO, Park J, Hegarty M, Jacques A, Eikelboom $\mathrm{RH}$, et al. Prevalence and risk factors for parent-reported recurrent otitis media during early childhood in the Western Australian Pregnancy Cohort (Raine) Study. J Paediatr Child Health. 2015; 51(4):403-409

3. Bennett K, Haggard M. Behaviour and cognitive outcomes from middle ear disease. Arch Dis Child. 1999; 80(1):28-35

4. Bennett K, Haggard M, Silva P, Stewart I. Behaviour and developmental effects of otitis media with effusion into the teens. Arch Dis Child. 2001; 85(2):91-95

5. Creps CL, Vernon-Feagans L. Infant Daycare and Otitis Media: Multiple Influences on Children's Later Development. J Appl Dev Psychol. 2000; 21(4):357378

6. Silva PA, Kirkland C, Simpson A, Steward IA, Williams SM. Some developmental and behavioral problems associated with bilateral otitis media with effusion. J Learn Disabil. 1982; 15(7)

7. Schilder AG, Van Manen JG, Zielhuis GA, Grievink EH, Peters SA, Van Den Broek P. Long-term effects of otitis media with effusion on language, reading and spelling. Clin Otolaryngol Allied Sci. 1993 Jun; 18(3):234-41 
8. Paradise JL. Otitis media during early life: how hazardous to development? A critical review of the evidence. Pediatr. 1981 Dec; 68(6):869-73

9. Teele DW, Klein JO, Rosner BA. Otitis media with effusion during the first three years of life and development of speech and language. Pediatr. 1984; 74(2):282-7

10. Paradise JL. Otitis media and child development: should we worry? Pediatr Infect Dis J. 1998; 17(11):1076-83

11. Rapin I. Conductive hearing loss effects on children's language and scholastic skills. A review of the literature. Ann Otol Rhinol Laryngol Suppl. 1979; 88(5 Pt 2 Suppl 60):3-12

12. Paradise JL, Rogers KD. On otitis media, child development, and tympanostomy tubes: new answers or old questions? Pediatr. 1986 Jan; 77(1):88-92

13. Roberts JE, Rosenfeld RM, Zeisel SA. Otitis Media and Speech and Language: A Meta-analysis of Prospective Studies. Pediatr. 2004; 113(3):e238-e248

14. Straker I. Mountain J, Jacques A, White S, Smith A, Landau, L, et al. Cohort Profile: The Western Australian Cohort (Raine) Study - Generation 2. Int. J. Epidemiol. 2017; 00(00):1-12

15. Brennan-Jones CG, Eikelboom RH, Jacques A, Swanepoel D, Atlas MD, Whitehouse AJO, et al. Protective benefit of predominant breastfeeding against otitis media may be limited to early childhood: results from a prospective birth cohort study. Clin Otolaryngol. 2016:1749-4486 
16. Robinson M, Oddy WH, Jianghong L, Kendall GE, de Klerk NH, Silburn SR, et al. Pre- and postnatal influences on preschool mental health: a large-scale cohort study. J Child Psychol Psychiatry. 2008; 49(10):1118-1128

17. Swanepoel DW, Eikelboom RH, Margolis RH. Tympanometry screening criteria in children ages 5-7 yr. J Am Acad Audiol. 2014; 25(10):927-36

18. Warnick EM, Bracken MB, Kasl S. Screening Efficiency of the Child Behavior Checklist and Strengths and Difficulties Questionnaire: A Systematic Review. Adolesc Ment Health 2008; 13(3):140-147

19. Knishkowy B, Palti H, Adler B, Tepper D. Effect of otitis media on development: a community-based study. Early Hum Dev. 1991; 26(2):101-111

20. Ruben RJ. A Time Frame of Critical/Sensitive Periods of Language Development. Acta Oto-Laryngologica. 1997; 117(2):202-205

21. Simpson SA, Thomas CL, van der Linden M, MacMillan H, van der Wouden JC, Butler CC. Identification of children in the first four years of life for early treatment for otitis media with effusion. Cochrane Database Syst Rev. 2007; 2007(1):1465-1858

22. Minter KR, Roberts JE, Hooper SR, Burchinal MR, Zeisel SA. Early Childhood Otitis Media in Relation to Children's Attention-Related Behavior in the First Six Years of Life. J Pediatr. 2001; 107(5):1037 
23. Bayer JK, Hiscock H, Ukoumunne OC, Price A, Wake M. Early childhood aetiology of mental health problems: a longitudinal population-based study. J Child Psychol Psychiatry. 2008; 49(11):1469-7610

24. Cabaj JL, McDonald SW, Tough SC. Early childhood risk and resilience factors for behavioural and emotional problems in middle childhood. BMC Pediatr. 2014; $14: 166$

25. Daly KA, Lindgren B, Giebink GS. Validity of parental report of a child's medical history in otitis media research. Am J Epidemiol. 1994; 139(11):1116-1121 